\title{
Impact of point A asymmetry on local control and survival for low dose-rate (LDR) brachytherapy in cervical cancer
}

\author{
Krisha J Opfermann, MD, Amy Wahlquist, MSc, John Watkins, MD, Matthew Kohler, MD, Joseph Jenrette III, MD \\ Radiation Oncology, Medical University of South Carolina, Charleston, USA
}

\begin{abstract}
Purpose: To evaluate whether Point A asymmetry in low dose-rate (LDR) brachytherapy is associated with local control (LC), disease-free survival (DFS) and/or overall survival (OS).

Material and methods: A retrospective analysis of disease control and survival outcomes was conducted for patients who underwent LDR brachytherapy for advanced cervical cancer. Institutional protocol entailed concurrent chemotherapy and whole pelvis radiotherapy (WPRT) over 5 weeks, followed by placement of Fletcher-Suit tandem and colpostat applicators at weeks 6 and 8. Objective Point A doses, 80-85 Gy, were accomplished by placement of Cesium-137 (Cs-137) sources. Cox proportional hazards regression models were used to assess associations between disease control and survival endpoints with variables of interest.

Results: The records of 50 patients with FIGO stage IB1-IVA cervical cancer undergoing LDR brachytherapy at our institution were identified. Thirty of these patients had asymmetry $>2.5 \%$, and 11 patients had asymmetry $>5 \%$. At a median survivor follow-up of 20.25 months, 15 patients had experienced disease failure (including 5 cervical/vaginal apex only failures and 2 failures encompassing the local site). Right/left dose asymmetry at Point A was associated with statistically significantly inferior LC $(p=0.035)$ and inferior DFS $(p=0.011)$ for patients with mean Point A dose of $>80$ Gy. Insufficient evidence existed to conclude an association with OS.

Conclusions: LDR brachytherapy may be associated with clinically significant dose asymmetry. The present study demonstrates that patients with Point A asymmetry have a higher risk of failure for DFS and LC.

Key words: brachytherapy, cervical, point A, Fletcher-Suit system.

\section{Purpose}

The first application of radium as intracavitary brachytherapy treatment of cancer of the uterus took place in 1908 [1]. Since its conception, brachytherapy has evolved as a staple modality of radiation delivery for treatment of cervical cancer. The localized radiation modality is often utilized to supplement (or "boost") the dose to the primary tumour during or following external beam treatment to the whole pelvis, while largely sparing the surrounding normal tissue. The LDR technique involves the identification and recording of doses to several reference "points", including bilateral Point A, Point B, a bladder point and a rectal point. Point $A$ is recognized as superior to the cervical os/flange by $2 \mathrm{~cm}$ and then lateral by $2 \mathrm{~cm}$. Point $B$ is representative of the dose to the obturator lymphatic region and is $2 \mathrm{~cm}$ superior and $5 \mathrm{~cm}$ lateral to the cervical os, in alignment with the body axis. With a $7 \mathrm{cc}$ Foley balloon in place, the posterior extent represents the bladder point and the rec- tal point is defined as $5 \mathrm{~mm}$ posterior to the vaginal packing at the level of the tandem and ovoid bisection [2].

Multiple LDR brachytherapy systems have been devised to maximize the therapeutic effect. The manual afterloading Fletcher-Suit tandem and colpostats system is used most commonly [2]. The Fletcher-Suit system may become displaced due to patient transfer, valsalva and/or organ movement $[3,4]$. Alternatively, midline placement technique may suffer in certain patients due to tumour bulk or disruption of anatomy following tumour response to chemotherapy and external beam radiotherapy, as well as suboptimal placement technique. The present investigation evaluates the impact of Point A dose symmetry on disease control and survival.

\section{Material and methods}

The institutional treatment protocol for early stage non-bulky cases (IA2-IB1) involved radiotherapy alone, 
while early stage bulky and loco-regionally advanced cases (IB2-IVA) are treated with concurrent chemoradiotherapy. Radiotherapy protocol included WPRT prescribed to a dose of $45 \mathrm{~Gy}$ in 25 once-daily fractions over the course of 5 weeks, followed by LDR brachytherapy boost in weeks 6 and 8 . Two radiopaque markers are then placed in the cervix to allow the visualization of the cervix on radiographs at the time of treatment planning. The uterus is sounded with a malleable probe to assess the tandem

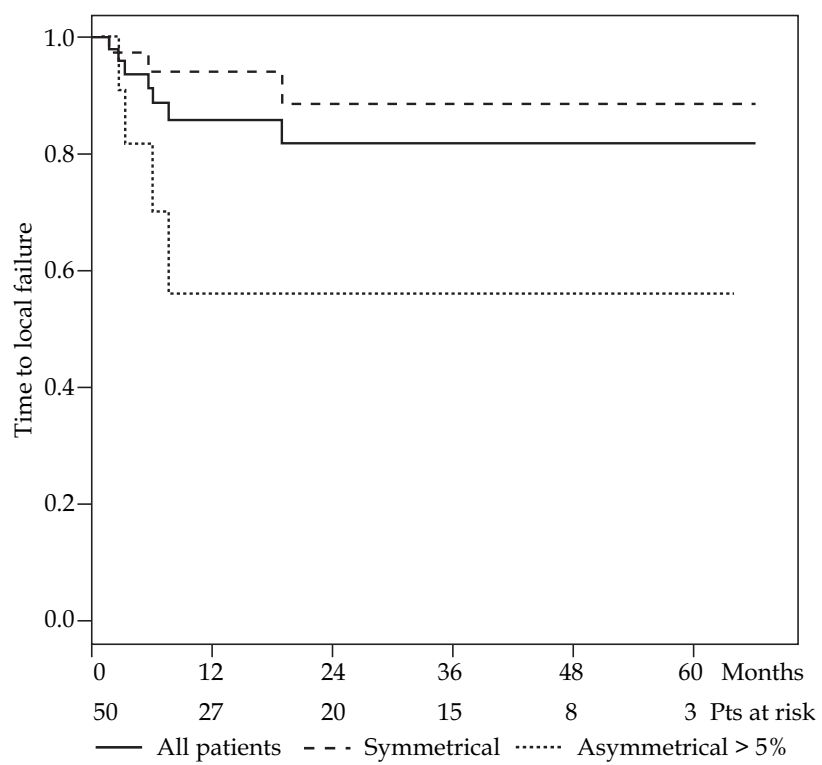

Fig. 1. LC survival curve: Kaplan-Meier curve demonstrating local control among all patients and patients with no significant asymmetry against patients with dose asymmetry $>5 \%$

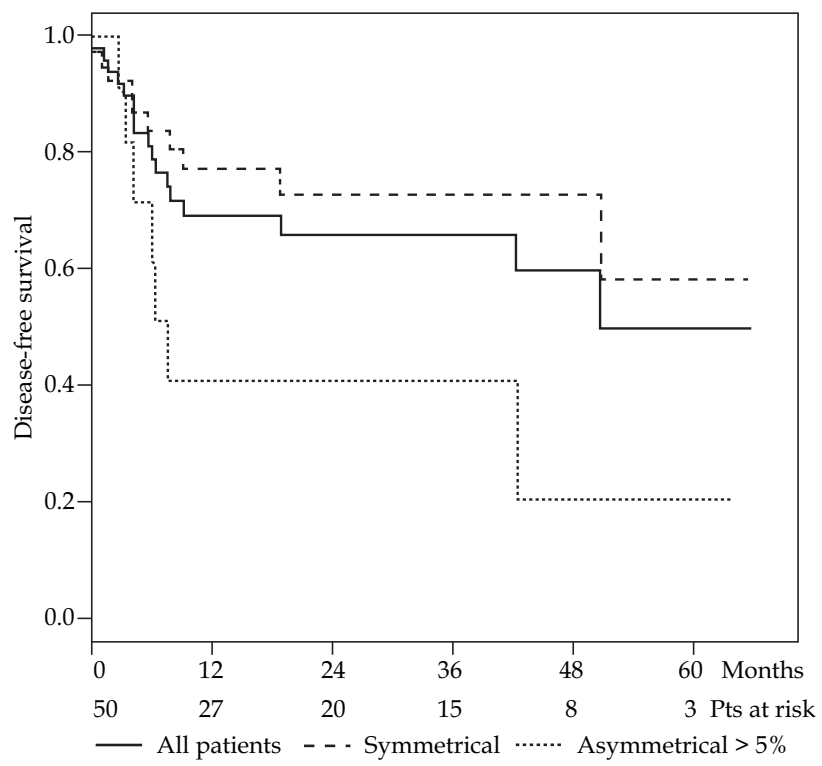

Fig. 2. DFS curve: Kaplan-Meier curve demonstrating disease-free survival among patients with no significant asymmetry versus patients with dose asymmetry $>5 \%$. The DFS curve for all patients combined is displayed as a solid line length and curvature. A Delcos Applicator Set tandem and ovoids, Best Medical International, Inc., is implanted with a gynecologic oncologist's assistance. After the tandem is satisfactorily placed (with or without real-time ultrasound guidance at the gynecologic oncologist's discretion), the largest diameter colpostat that can be accommodated is inserted into the vaginal fornices bilaterally. Plain radiographic films are obtained in order to assess the midline position of the tandem and flange placement.

After the position of the device is confirmed satisfactory, dosimetric points of interest (including Point A, Point B, a bladder point and a rectal point) are labeled in order to guide treatment planning. Using standard loading techniques, the dose distribution assumes a pear-shaped high dose region that concentrates dose at the cervix. Two LDR brachytherapy administrations are employed to deliver a total dose to Point A of 80-85 Gy. Typically, the first LDR administration delivers approximately two-thirds of the boost dose and the second completes the course of therapy. Once a satisfactory plan has been determined, the sources are loaded with Cs-137 sources at the patient's bedside.

Following Institutional Review Board (IRB) approval at the Medical University of South Carolina (MUSC), a retrospective research database was created with study-specific patient, tumour, treatment and outcome data fields. Eligible cases were identified by a review of the departmental quality assurance database, medical records and office management software for those patients who had undertaken placement of the Fletcher-Suit intracavitary applicator for LDR brachytherapy. Selection criteria for the study included all FIGO stage IB1-IV [5] cervical cancer patients who underwent intrauterine tandem and colpostat placement as detailed previously. Dose delivered as recorded at Point $A$, left $\left(A_{L}\right)$ and Point $A$, right $\left(A_{R}\right)$ was documented from a review of dosimetry plans. Asymmetry of dose distribution was determined by division of the difference between $A_{R}$ and $A_{L}\left(A_{R}-A_{L}\right)$ by the mean dose to Point A $\left(\mathrm{A}_{\mathrm{Ave}}\right)$.

The timing and location of initial disease recurrence (earliest sign of clinical, radiographic or pathologic disease), was determined from a review of patient charts. Recurrence was scored as: local, regional, local/regional with distant or distant alone. Endpoints included LC, DFS and OS were measured from the date of final brachytherapy implant to the date of death (OS), recurrence from any site or death (DFS), recurrence encompassing the local site (LC) or date of last follow-up.

\section{Statistical analysis}

The Kaplan-Meier curves for LC (Fig. 1), DFS (Fig. 2) and OS curves (Fig. 3) were generated using the statistical software: $\mathrm{R}$ version 2.10.1. The time to event outcomes for $\mathrm{LC}$, DFS and OS were calculated from the date of final brachytherapy implant to date of event of interest or last follow-up for patients with no event. Univariate analysis of patient-, tumour-, and treatment-associated factors to determine association with disease control and survival endpoints were performed using Cox proportional hazards regression models for continuous and bivariate categori- 


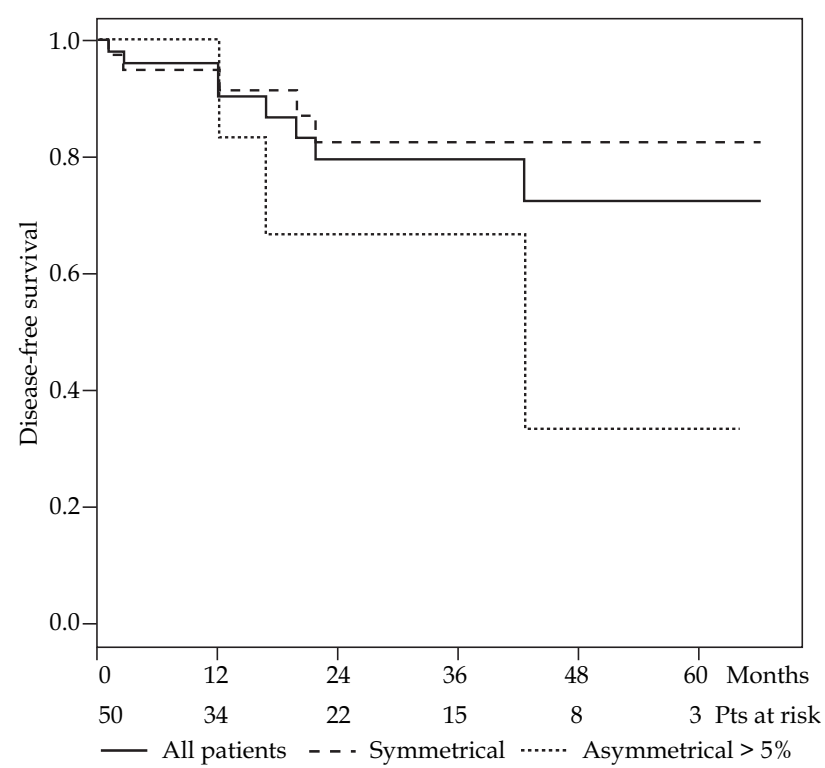

Fig. 3. OS curve: Kaplan-Meier curve demonstrating overall survival among patients with no significant asymmetry versus patients with dose asymmetry $>5 \%$. The OS curve for all patients combined is displayed as a solid line

cal factors [6]. The proportional hazards assumption was tested and assumed to be valid for $p \geq 0.05$. Statistical significance was assessed at the $\alpha=0.05$ level for all testing.

\section{Results}

We identified the complete medical and dosimetry records of 50 patients who underwent LDR treatment at our institution from 1996-2008 with FIGO stage IB1-IVA cervical cancer (Table 1). At the completion of EBRT, a boost was added with 1 LDR Fletcher-Suit implant in 7 patients and 2 consecutive implants in 43 other patients. Asymmetry was determined as asymmetry of the total dose from pelvic radiation and 2 consecutive implants ( $\left.\mathrm{Pt} \mathrm{A}_{\text {Rtotal }}-\mathrm{Pt} \mathrm{A}_{\text {Ltotal }}\right)$ / Pt $\mathrm{A}_{\text {ave }}$, where Pt $A_{\text {ave }}$ is the average total dose to Point $\mathrm{A}$.

Forty patients had an average Point $A$ dose $\geq 80$ Gy (these patients were evaluated during the subset analysis). Eleven of the original 50 patients had an asymmetry $>5 \%$, respectively. Patients whose dose asymmetry represented $>5 \%$, were further analyzed in relation to patients with relatively symmetrical implants (asymmetry $\leq 5 \%$ of the brachytherapy dose delivered).

At a median survivor follow-up of 20.3 months (range, 3-65.8), 15 patients had experienced disease failure with a component of local failure. Last follow-up was determined based on time of last clinical assessment with physical examination with the patient's Radiation Oncologist/Gynecology Oncologist. Eight patients had died (6 of or with recurrent local disease). Seven of the 15 patients with disease failure had experienced disease failure at the local site (5 isolated local failures and 2 patients with multiple sites of disease at the time of diagnosed recurrence, incorporating local/regional failure). Table 2 details sites of recurrence in the 7 patients that developed a local/regional recurrence alone or in combination with distant disease.
Table 1. Patient characteristics and treatment details of all patients reviewed

\begin{tabular}{|c|c|c|}
\hline Variables & Values & $\begin{array}{l}\text { Number } \\
(n=50)\end{array}$ \\
\hline Age (years) & median & $50.5(30-88)$ \\
\hline Age distribution & $\begin{array}{l}\geq 60 \text { yrs } \\
<60 \text { yrs }\end{array}$ & $\begin{array}{l}14(28 \%) \\
36(72 \%)\end{array}$ \\
\hline Race & $\begin{array}{l}\text { Caucasian } \\
\text { other }\end{array}$ & $\begin{array}{l}24(48 \%) \\
26(52 \%)\end{array}$ \\
\hline $\begin{array}{l}\text { FIGO Stage } \\
\text { (AJCC 6th ed.) }\end{array}$ & $\begin{array}{l}\text { IB1 } \\
\text { IB2 } \\
\text { IIA } \\
\text { IIB } \\
\text { IIIA } \\
\text { IIIB } \\
\text { IVA }\end{array}$ & $\begin{array}{c}6(12 \%) \\
13(26 \%) \\
5(10 \%) \\
8(16 \%) \\
0(0 \%) \\
14(28 \%) \\
4(8 \%)\end{array}$ \\
\hline Histology & $\begin{array}{l}\text { squamous cell } \\
\text { carcinoma } \\
\text { adenocarcinoma }\end{array}$ & $\begin{array}{c}43(86 \%) \\
7(14 \%)\end{array}$ \\
\hline WBRT (Gy) & median & $45(41.4-55)$ \\
\hline EBRT boost & $\begin{array}{l}\text { yes } \\
\text { no }\end{array}$ & $\begin{array}{r}7(14 \%) \\
43(86 \%)\end{array}$ \\
\hline 1 tandem/2 ovoids & $\begin{array}{l}\text { implant \#1 } \\
\text { implant \#2 } \\
(n=43)\end{array}$ & $\begin{array}{l}46(92 \%) \\
38(86 \%)\end{array}$ \\
\hline $\begin{array}{l}\text { Median } \\
\text { duration (hrs) }\end{array}$ & $\begin{array}{l}\text { implant \#1 } \\
\text { implant \#2 }\end{array}$ & $\begin{array}{l}48(19.9-72) \\
24(17-65)\end{array}$ \\
\hline $\begin{array}{l}\text { Brachytherapy } \\
\text { dose (Gy) } \\
\text { - per implant }\end{array}$ & $\begin{array}{l}\text { Pt } A_{R 1} \\
\text { Pt } A_{L 1} \\
\text { Pt } A_{R 2} \\
\text { Pt } A_{L 2}\end{array}$ & $\begin{array}{l}26.13(11.08-40.62) \\
24.52(9.67-39.38) \\
13.44(5.18-30.29) \\
13.16(2.65-28.67)\end{array}$ \\
\hline $\begin{array}{l}\text { Brachytherapy } \\
\text { Dose (Gy) - total }\end{array}$ & $\begin{array}{l}\text { Pt A } \\
\text { Pt Atotal } \\
\text { Ltotal }\end{array}$ & $\begin{array}{l}84.3(65.63-88.49) \\
83.12(65.46-90.92)\end{array}$ \\
\hline Asymmetry & $\begin{array}{l}>2.5 \% \\
>5 \%\end{array}$ & $\begin{array}{l}30(60 \%) \\
11(22 \%)\end{array}$ \\
\hline
\end{tabular}

WBRT - Whole Breast Radiotherapy, EBRT - External Beam Radiotherapy

For the subset of 40 patients who received a therapeutic dose of radiation ( $\geq 80$ Gy to Point $A_{\text {ave }}$ ), Cox proportional hazard models showed statistically significant relationships between dose asymmetry and LC $(p=0.035)$, as well as between dose asymmetry and DFS $(p=0.011)$. The 1-year survival estimates (and 95\% confidence interval) for LC in the asymmetrical group was 50.0\% (20.4$100 \%)$, compared to $92.5 \%(82.9-100 \%)$ in the symmetrical group. For DFS, the 1-year estimates (95\% CIs) were $30.0 \%(9.5-94.3 \%)$ and $72.0 \%(57.1-90.9 \%)$ for asymmetry and symmetry groups, respectively. There was insufficient evidence to establish an association of Point A asymmetry with OS. Six patients, for reasons not recorded, never received a second implant. One patient had a premature removal of the implant \#2, 2 patients had a total dose slightly under $80 \mathrm{~Gy}$ and 1 patient was recorded as having "ovoids that were never loaded due to asymmetry".

Cox proportional hazards models were used to determine if there was a significant relationship between FIGO stage, length of treatment and concurrent chemotherapy with any of the outcomes of interest (LC, DFS, OS) on uni- 
Table 2. Patients experiencing a component of local failure: type of disease failure and specific location detailed

\begin{tabular}{lll}
\hline Patient & $\begin{array}{l}\text { Type } \\
\text { of recurrence* }\end{array}$ & $\begin{array}{l}\text { Location } \\
\text { of recurrence }\end{array}$ \\
\hline 1 & local & cervix \\
\hline 2 & local & cervix with extension into the colon \\
\hline 3 & local & vaginal apex \\
\hline $4^{* *}$ & local & cervix \\
\hline $5^{* *}$ & local & cervix \\
\hline $6^{* *}$ & $\begin{array}{l}\text { local-regional } \\
\text { \& distant }\end{array}$ & $\begin{array}{l}\text { perineal/labia majoria lesion, } \\
\text { mass posterior and lateral to } \\
\text { the bladder, pelvic lymph nodes, } \\
\text { umbilical mass, pulmonary hilum, } \\
\text { liver, subcutaneous abdominal fat }\end{array}$ \\
\hline 7 & local-regional & cervix and pelvic nodes \\
& $\&$ distant &
\end{tabular}

*The other 8 patients that developed disease failure did not have local disease present at the time of treatment failure and as such are not detailed in the Table above

**Patients classified as "asymmetrical implants". In addition, 3 patients with asymmetrical implants did not experience disease recurrence and 2 developed distant failure as the first site of failure

variate analysis. However, no statistically significant association was identified with any of these factors.

These models were also adjusted for asymmetry to determine whether the combination with asymmetry and FIGO stage, length of treatment or concurrent chemotherapy was related to any of the outcomes. There was no association noted between asymmetry (imbalance $>5 \%$ for $\geq 80$ Gy Ave Point A dose) and LC, DFS or OS when adjusting for FIGO stage. There was also no association between asymmetry and LC, DFS or OS when adjusting the length of treatment time. When adjusting for concurrent chemotherapy, however, there were significant associations between asymmetry and OS $(p=0.036)$, DFS $(p=0.0091)$ and time to local failure $(p=0.035)$. The hazard risk of local failure occurring is 11.6 times higher for those with asymmetry compared to those without. The risk of a death or lo$\mathrm{cal} /$ distant recurrence is 4.6 times higher for those with asymmetry compared to those without. The overall risk of death is 6.9 times higher for those with asymmetry compared to those without.

\section{Discussion}

The rapid fall-off due to attenuation and distancing of the radiation during brachytherapy allows sparing of surrounding normal tissue. The present study analyses the Fletcher-Suit LDR loading system, its final plan dosimetry and impact on patient outcome. The loading system has a tandem device fixed in the uterus and 2 colpostats at each vaginal fornix to fixate the target and provide a pear-shape region of dose conformal to the local anatomy. Variations in dose distribution to target Point $\mathrm{A}$ on the right versus the left may come about due to patient anatomy, tumour mass or from improper insertion/immobilization of the loading equipment. Anatomic distortions that may concern a change in uniform dose to Point A include lesions restricted primarily to one cervical lip, massive cervical lesions expanding the cervix or isthmus, vaginal tumour involvement and parametria shortening with its associated tandem angulation [7]. We studied 50 patients at our institution with FIGO stage IB1-IVA cervical cancer treated with EBRT and 1-2 sessions LDR brachytherapy to find 11 cases $(22 \%)$ having resulted in asymmetry of $>5 \%$ of dose.

The uterus may assume a position of anteversion or retroversion (angle alpha) and it may also exhibit a right or left shift to the midline (angle beta), which results in asymmetry of the system. This was examined by Vedula for the flexible Amersham system since this system assumes the anatomy of the uterus [8]. In particular, dose to Point A was examined as a reference point since it is believed to represent a region of radiosensitivity in the parametrium, where the uterine artery and the ureter cross. In this study of 94 cases, asymmetry of up to $10 \%$ at Point A was found in $93.6 \%$ of cases. In $20.2 \%$ of cases showed a $\geq 20 \%$ difference between the specified and the mean Point A dose rates. Higher doses were also noted at the rectum and bladder in reference to a change in the parameters of the angle beta. For the rigid system applicators, the uterine geometry assumes that of the applicator and beta is assumed to be $0^{\circ}$. Identical Point $A$ positions are defined and the Point $\mathrm{A}$ dose rates on the right and left are assumed equal [8]. In a "classic" Fletcher application, the tandem axis bisects the height of it and is equidistant from the colpostats and the most distal end of the cervical source minimally overlaps the cephalad rim of the colpostats to optimize dose distribution to the cervix, vagina and parametria [7].

Fox Chase addressed the concern of ideal LDR placement and patient outcome. Their study assessed the distance between the right colpostat source/left colpostat source and the distal tandem source and the symmetry of the colpostat placement. Implants were "ideal" only when all 3 parameters were deemed satisfactory. Significantly improved 5 -year LC was seen when comparing ideal and adequate placements to unacceptable placements $(68 \%$ vs. $34 \%$, $p=0.02)$. A strong trend was also noted toward improved 5 -year survival among the group with ideal and adequate implants as opposed to unacceptable implants (60\% vs. $40 \%$ ). Multivariate analysis showed that the technical adequacy of the brachytherapy implant was the most important prognostic discriminator. In 66 patients, a series of models was considered for each of the previously identified prognostic factors: overall treatment time, age, Karnofsky performance status (KPS), stage and total dose. In their patient cohort there were a total of 29 local failures (4 in the ideal group, 14 in the adequate group and 11 in the unacceptable group). In multivariate analysis of LC in individual relationship with each of the previously identified prognostic factors listed above, technical placement was found to be significant. This finding leads to concern that the worsening of LC was attributed to the performance of unacceptable implants that may reflect regions of underdosage ("cold spots") within the target volume [9]. Within the present study of 50 patients, there were only a total of 5 confined local failures and 2 cases where a local/regional failure was a component of the discovered disease recurrence at patient follow-up. Despite the low number of disease fail- 
ures, our study also revealed an agreement in patients treated to adequate dose between local recurrence/local-regional and distal recurrence and Point A asymmetry and a significant relationship between DFS and Point A asymmetry.

\section{Conclusions}

One might theorize that the likelihood of an implant asymmetry might be associated with increased tumour burden, due to distortion of anatomy or inadequacy of implant placement due to tumour bulk and thus the increased risk of tumour failure, reflects a tumour-related factor rather than a technical aspect of implant placement. That being stated, the present study did not identify any association of FIGO stage with disease control endpoints, when substratified by implant asymmetry. To our knowledge, this is the first investigation to demonstrate such a finding and this would argue for the importance of implant symmetry in all clinical situations, punctuated by the findings of adequately dosed implants (to $\geq 80 \mathrm{~Gy}$ at Point A). Another consideration, however, is that in cases of asymmetrical tumour burden, a purposely adapted asymmetrical dose distribution may in fact be favorable to provide adequate tumour coverage. Such a situation and system would be best facilitated by more detailed image guidance and/or HDR brachytherapy [10].

In conclusion, standard LDR methods result in Point A asymmetry of dosing, thus predisposing to an underdosing of critical structures. Our study demonstrates a possible association between Point A asymmetry and DFS and LC, particularly in patients with an average Point A dose $\geq 80 \mathrm{~Gy}$.

\section{References}

1. Khan FM. The physics of radiation therapy. Lippincott Williams \& Wilkins, Philadelphia 2010; 343-346.

2. Nag S, Chao C, Erickson B et al. The American brachytherapy society recommendations for low-dose-rate brachytherapy for carcinoma of the cervix. Int J Radiat Oncol Biol Phys 2002; 52: 33-48.

3. Prabhakar R, Jagadesan P, Haresh KP et al. A study on the variation of bladder and rectal doses with respiration in intracavitary brachytherapy for cervix cancer. J Contemp Brachyther 2010; 2: 24-27.

4. Koushik K, Bilimagga R, Rao N et al. Positional variation of applicators during low dose rate intracavitary brachytherapy for cervical cancer: a prospective study. J Contemp Brachyther 2010; 2: 93-97.

5. Greene FL, Page DL, Fleming ID et al. AJCC cancer staging manual sixth edition. Springer, Chicago 2002; 269-262.

6. Cox DR. Regression Models and Life-Table. Journal of the Royal Statistical Society. Series B (Methodological) 1972; 34: 187-220.

7. Potish RA. The effect of applicator geometry on dose specification in cervical cancer. Int J Radiat Oncol Biol Phys 1990; 18: 1513-1520.

8. Vedula R. Implication of uterine configuration on dosimetry to point $\mathrm{A}$ using the amersham afterloading system. Int $\mathrm{J} R a$ diat Oncol Biol Phys 1993; 25: 721-731.

9. Corn BW, Hanlon AL, Pajak TF et al. Technically accurate intracavitary insertions improve pelvic control and survival among patients with locally advanced carcinoma of the uterine cervix. Gynecol Oncol 1994; 53: 294-300.

10. Esthappan J, Ma DJ, Narra VR et al. Comparison of apparent diffusion coefficient maps to T2-weighted images for target delineation in cervix cancer brachytherapy. J Contemp Brachyther 2011; 3: 193-198. 